Ferrata Storti Foundation

\title{
Meningioma 1 is indispensable for mixed lineage leukemia-rearranged acute myeloid leukemia
}

\author{
Amit Sharma, ${ }^{1}$ Nidhi Jyotsana,,${ }^{1}$ Razif Gabdoulline, ${ }^{1}$ Dirk Heckl, ${ }^{2}$ \\ Florian Kuchenbauer, ${ }^{3}$ Robert K. Slany, ${ }^{4}$ Arnold Ganser ${ }^{1}$ and Michael Heuser ${ }^{1}$
}

${ }^{1}$ Department of Hematology, Hemostasis, Oncology and Stem Cell Transplantation, Hannover Medical School, Hannover, Germany; ${ }^{2}$ Department of Pediatric Hematology and Oncology, Hannover Medical School, Hannover, Germany; ${ }^{3}$ British Columbia Cancer

Haematologica 2020

Volume 105(5):1294-1305 Agency, Vancouver, British Columbia, Canada and ${ }^{4}$ Department of Genetics, FriedrichAlexander-University Erlangen-Nürnberg, Erlangen, Germany

\section{ABSTRACT}

M ixed lineage leukemia (MLL/KMT2A) rearrangements (MLL-r) are one of the most frequent chromosomal aberrations in acute myeloid leukemia. We evaluated the function of Meningioma 1 (MN1), a co-factor of HOXA9 and MEIS1, in human and murine MLLrearranged leukemia by CRISPR-Cas9 mediated deletion of MN1. MN1 was required for in vivo leukemogenicity of MLL positive murine and human leukemia cells. Loss of MN1 inhibited cell cycle and proliferation, promoted apoptosis and induced differentiation of MLL-rearranged cells. Expression analysis and chromatin immunoprecipitation with sequencing from previously reported data sets demonstrated that MN1 primarily maintains active transcription of HOXA9 and HOXA10, which are critical downstream genes of MLL, and their target genes like BCL2, MCL1 and Survivin. Treatment of MLL-rearranged primary leukemia cells with antiMN1 siRNA significantly reduced their clonogenic potential in contrast to normal $\mathrm{CD} 4^{+}$hematopoietic progenitor cells, suggesting a therapeutic window for $\mathrm{MN1}$ targeting. In summary, our findings demonstrate that MN1 plays an essential role in MLL fusion leukemias and serve as a therapeutic target in MLL-rearranged acute myeloid leukemia.

Received: November 6, 2018.

Accepted: August 8, 2019.

Pre-published: August 14, 2019.

doi:10.3324/haematol.2018.211201

Check the online version for the most updated information on this article, online supplements, and information on authorship \& disclosures: www.haematologica.org/content/105/5/1294

(C)2020 Ferrata Storti Foundation

Material published in Haematologica is covered by copyright. All rights are reserved to the Ferrata Storti Foundation. Use of published material is allowed under the following terms and conditions:

https://creativecommons.org/licenses/by-nc/4.0/legalcode. Copies of published material are allowed for personal or internal use. Sharing published material for non-commercial purposes is subject to the following conditions: https://creativecommons.org/licenses/by-nc/4.0/legalcode, sect. 3. Reproducing and sharing published material for commercial purposes is not allowed without permission in writing from the publisher.

\section{Introduction}

Acute myeloid leukemia (AML) is associated with a plethora of genetic alterations such as chromosomal rearrangements and mutations, ${ }^{1}$ with some of them being generic for different types of leukemias and cancers. ${ }^{1}$ MLL1 (Mixed lineage leukemia $1 /$ KMT2A) rearrangements are one such example that are found in myeloid as well as lymphoid leukemias. ${ }^{2}$ Approximately 135 different MLL rearrangements have been identified so far, but only nine specific gene fusions (including AF4, AF9, ENL, and AF6) account for more than $90 \%$ of all oncogenic recombinations. ${ }^{3,4}$ A unifying hallmark of all MLL-rearranged (MLL-r) leukemias is the deregulation of clustered HOXA/MEIS1 genes. ${ }^{2}$ Transcriptional activation of MLL target genes (HOXA9/MEIS1) is associated with an increase in histone H3 lysine79 dimethylation (H3K79me2) across the respective gene locus, which is specifically mediated by histone methyltransferase DOT1., Recently, several studies in patients and murine models have highlighted the importance of co-operating genetic alterations in MLLr leukemia progression. In 40-50\% of MLL-r AML cases, RAS and FLT3 mutations have been shown to accelerate leukemogenesis, and Mn1, Bcl11a and Fosb have been identified as co-operating oncogenes in a murine leukemia virus insertional mutagenesis model. ${ }^{4,6}$

MN1 (Meningioma-1) is frequently over-expressed in AML patients and is associated with a poor prognosis. ${ }^{7-13}$ However, in patients with inv(16), highest $M N 1$ expression has been reported with favorable prognosis to current therapeutics. ${ }^{11} \mathrm{MN} 1$ functions as a transcriptional regulator that co-operates with the nuclear receptors for retinoic acid (RAR) and vitamin D, by acting as co-activator or co-repressor, depending on the interacting partners. ${ }^{14-16}$ In addition, $M N 1$ is frequently over-expressed and occasionally fused to TEL as part of the rare MN1-TEL translocation. ${ }^{17} \mathrm{Mn} 1$ is known 
to be co-operating partner of several oncogenic fusion genes (NUP98-HOXD13, ${ }^{18}$ CALM-AF10, ${ }^{19} \mathrm{MLL}^{2}-\mathrm{AF9}^{6}$ and MLL-ENL) ${ }^{20}$ and mutated RUNX1, ${ }^{21}$ and as a common target of insertional mutagenesis in a hematopoietic stem cell (HSC) gene therapy trial, ${ }^{22}$ thereby promoting leukemogenesis.

Interestingly, MN1-induced AML is also dependent on Hoxa cluster genes and Meis1. ${ }^{23}$ Multipotent progenitor cells (MPP) and common myeloid progenitors (CMP) have been identified as the cell of origin in MN1-induced AML, while granulocyte-macrophage progenitors (GMP) cannot be transformed. ${ }^{23}$ We found that the differential expression of Hoxa9, Hoxa10 and Meis1 in MPP/CMP compared to GMP cells was responsible for the ability of MN1 to transform the more immature, but not the more mature, progenitor cells. ${ }^{23}$ One important difference between MN1 and MLL-r leukemia is that MN1 cannot activate Hox gene expression by itself, while MLL-AF9 can. ${ }^{23,24}$ Therefore, MN1 is unable to transform GMP cells, while MLL-AF9 can transform myeloid progenitor cells down to the differentiation state of a GMP. Both MLL-AF9- and MN1-induced leukemias depend on the H3K79 methyltransferase DOT1L. ${ }^{14,25,} 26$ In addition, deletion of M1l and Dot1l in MN1-expressing cells abrogated the cell of origin-derived gene expression program, including the expression of Hoxa cluster genes, and impaired the leukemogenic activity of MN1 in vivo. ${ }^{14}$ However, it is not known whether MLL-AF9 transformed cells also depend on MN1.

High MN1 expression confers resistance to all-trans retinoic acid (ATRA)-induced differentiation and chemotherapy-induced cytotoxicity. ${ }^{7,27}$ Recent studies have shown that pyrimethamine [a dihydrofolate reductase (DHFR) inhibitor] and DOT1L inhibitors possess antileukemic effects in MN1 ${ }^{\text {hi }}$ AML cells. ${ }^{14,27}$ However, the mechanism of MN1-induced AML and drug resistance is still not completely understood due to its little structural/functional similarity to any other protein. ${ }^{14} \mathrm{Mn} 1$ null mice have severe defects in bones of the cranial skeleton, yet the effects of its deletion in hematopoiesis/leukemia are not known. ${ }^{28}$ Here, we show that CRISPR-Cas9-mediated deletion of MN1 in MLL-r leukemias, and consequently treatment of MLL-r leukemias with an anti-MN1 siRNA, led to strong anti-leukemic effects, including increased terminal myeloid differentiation and suppression of leukemic growth in vitro and in vivo.

\section{Methods}

\section{Viral vectors, vector production, and CRISPR}

Briefly, helper-free recombinant retrovirus harvested from supernatants of the transfected ecotropic Phoenix packaging cell line was used to transduce 5-fluoro-uracil treated mouse bone marrow cells to generate an immortalized MLL-AF9 cell line, as described before. $^{15,29}$ CRISPR lentiviral vector L40C-CRISPR.EFS.dTomato (Addgene \# 89392) was kindly provided by Dr. Dirk Heckl, Hannover Medical School, Hannover, Germany. ${ }^{30}$ CRISPR-Cas9 target sites in the Mn1/MN1 gene were selected using the CCTop selection tool. ${ }^{31}$ Single guide RNA (sgRNA) were cloned in the CRISPR-Cas9 vector. The list of sgRNA is summarized in Online Supplementary Table S1.

\section{Cell lines and primary cells}

Bone marrow from healthy donors was obtained from the transplantation unit of Hannover Medical School. Mononuclear cells from peripheral blood or bone marrow were isolated by density gradient centrifugation. $\mathrm{CD} 34^{+}$cells were isolated using the CD34 microbead kit (Miltenyi Biotech, Bergisch Gladbach, Germany), as per manufacturer's protocol. AML patient samples were taken from the AML-myelodysplastic syndromes (AMLMDS) repository of Hannover Medical School. Written informed consent was obtained for the use of patient samples in accordance with the Declaration of Helsinki, and the study was approved by the institutional review board of Hannover Medical School (ethical vote 1187-2011).

\section{Mouse transplantation and homing assay}

NOD-SCID and NOD/SCID/IL2rgnull (NSG) mice were bred and maintained in pathogen-free conditions in the animal laboratory of Hannover Medical School, Hannover, Germany. All animal experiments were approved by the Lower Saxony state office for consumer protection, Oldenburg, Germany.

\section{siRNA and lipid nanoparticles}

All siRNAs (MN1 and control/AHA1) were purchased from Axolabs GmbH (Kulmbach, Germany). siRNAs were packaged in lipid nanoparticles (LNP), as described previously. ${ }^{32}$

\section{Gene expression and chromatin immunoprecipitation sequencing analysis}

We performed gene expression profiling of in vitro cultured MLL-AF9/Mn1wt versus MLL-AF9/Mn1null cells in triplicate. RNA was extracted using the standard trizol method and was further used for gene expression profiling. Gene expression profiling using extracted RNA from MLL-AF9/MNn1wt and MLLAF9/MNn1null cells was performed on Affymetrix GeneChip Mouse 4302.0 arrays (43,000 probes). The whole dataset can be found at GEO (GSE130631) for public access.

Chromatin immunoprecipitation sequencing (Chip-Seq) DNA binding data were taken for H3K79me2 from GSE55038, ${ }^{33}$ MLLAF9 from GSE29130, ${ }^{25}$ Hoxa9 from GSE33518, ${ }^{34}$ and MN1 and MEIS1 from our previous publication. ${ }^{23}$

\section{Statistical analysis}

Pairwise comparisons were performed by Student t-test for continuous variables. Two-sided significance was set at $P<0.05$. Comparison of survival curves were performed using the log rank test. Statistical analyses were performed with Microsoft Excel (Microsoft, Munich, Germany) and GraphPad Prism 5 (GraphPad Software, La Jolla, CA, USA).

More detailed information on the materials and methods used can be found in the Online Supplementary Appendix.

\section{Results}

MLL-AF9 cell proliferation depends on Meningioma 1

To study the effects of MN1 gene inactivation in leukemia cells, we generated CRISPR-Cas9 engineered MN1null murine and human leukemia cells and characterized these cells in vitro and in vivo (Figure 1A). MN1 was deleted in murine cells transformed by MLL-AF9, HOXA9, HOXA9MEIS1, E2A-HLF and 10 human leukemia cell lines: THP-1, MV-4-11, NB4, OCI-AML2, OCI-AML3, U937, K562, Kasumi-1, HL-60 and HEL. Ninety-six to 288 transduced cells per cell line were single cell sorted and the outgrowing clones were evaluated for MN1 deletion by qualitative RT-PCR (qRT-PCR), western blot and sequencing (Online Supplementary Tables S2 and $S 3$ and Online Supplementary Figure S1A-F). While up to 
$30 \%$ of the single sorted cells grew out as clones, only 0 $8.9 \%$ of the single sorted clones were MN1-deleted, and only one MN1-deleted clone was obtained from four different murine cell lines with HOX-dependent oncogene expression (Online Supplementary Table S2), suggesting that MN1 loss impairs clonogenicity of MLL-r and HOXA9transformed cells. Subsequently, in parallel to MLL-AF9 mouse cells, we also studied THP-1 and MV-4-11 (positive for MLL-AF9 and MLL-AF4, respectively) and the nonMLL-r human cell line U937. Loss of MN1 more potently reduced proliferation in MLL-r cells as compared to nonMLL-r leukemia cells (Figure 1B-D and Online Supplementary Figure S2A). The inhibitory effect of MN1 loss in U937 cells may be due to high HOXA9 and MEIS1 expression (Online Supplementary Figure S2B and C). The cell lines K562, Kasumi-1 and HL-60 had the lowest HOXA9 and MEIS1 expression levels, but had the highest rate of MN1-deleted clones (Online Supplementary Table S2), indicating a dependence on MN1 in MLL-r and HOXA9/MEIS1 expressing cells.

$\mathrm{MN1}$ deletion drastically impaired the colony-forming potential up to 22-fold and reduced the size of the colonies in MLL-AF9 transformed murine cells (Figure 1E and 1F). Due to potential unpredictable off-target effects, we tested one additional sgRNA (sgRNA-4; positioned at a different location), which had a similar effect as the initial sgRNA (Online Supplementary Figure S3A and B). Thus, in vitro proliferation and colony-forming potential of MLL-rearranged cells require $\mathrm{MN1}$ expression both in murine and human leukemia cells.

A

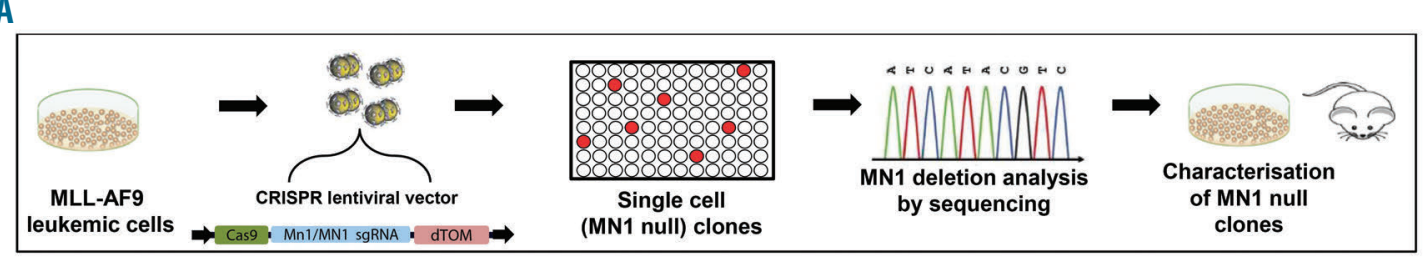

B

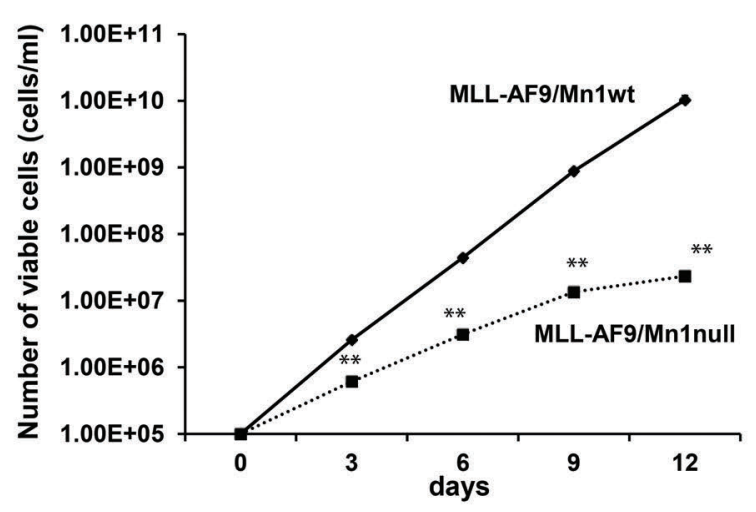

D

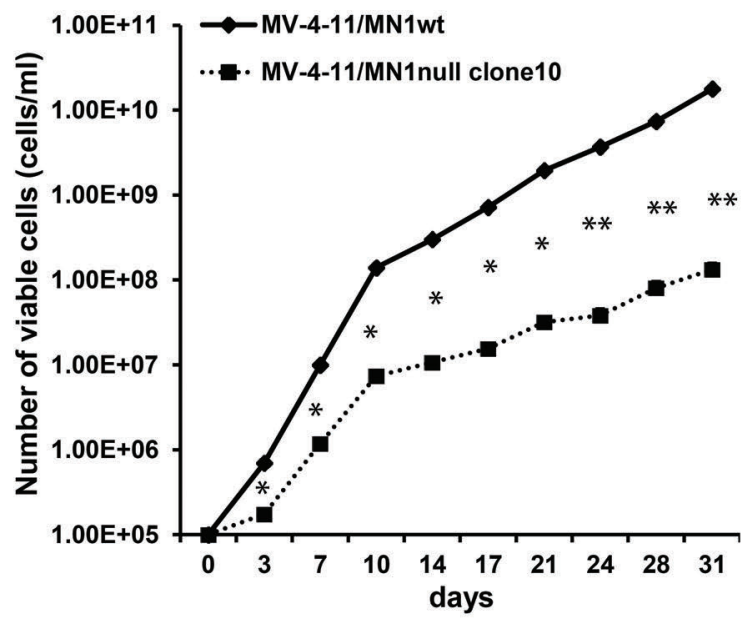

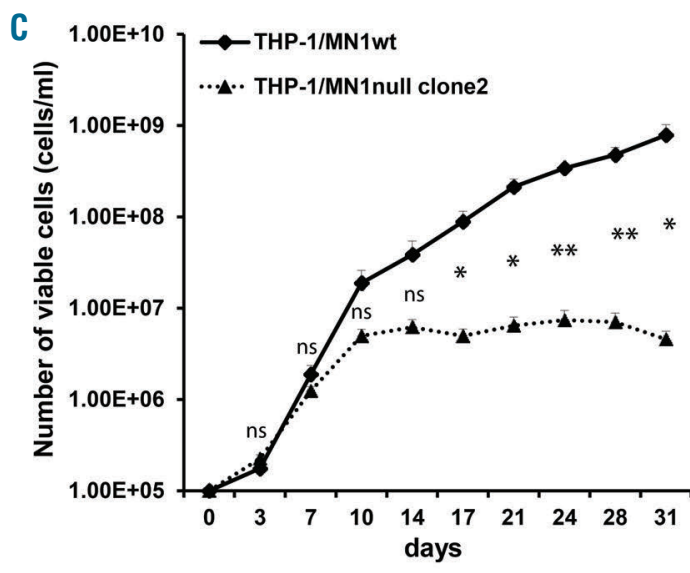

$\mathbf{E}$

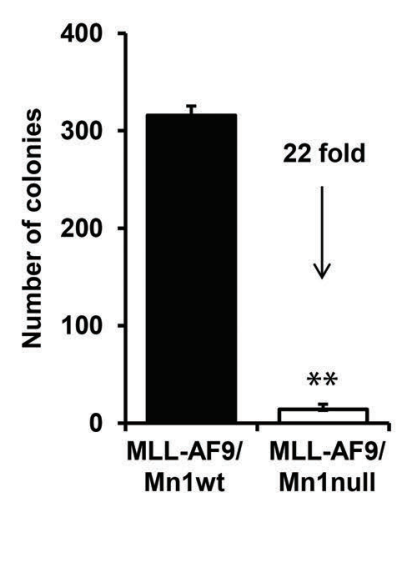

MLL-AF9/Mn1wt

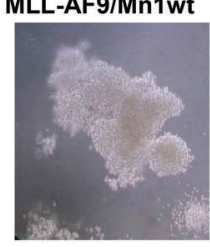

MLL-AF9/Mn1null

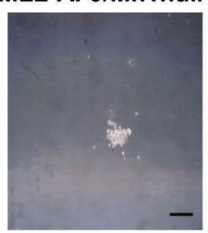

Figure 1. MLL-AF9 cell proliferation depends on Meningioma 1 (MN1). (A) Schematic outline of the strategy for deletion of MN1/Mn1 in murine and human cell lines. (B) Cumulative cell counts of MLL-AF9/Mn1wt and MLL-AF9/Mn1null cells [mean \pm standard error of mean (SEM), $n=3$ ]. (C) Cumulative cell counts of THP-1 MN1wt and MN1null cells (mean \pm SEM, $n=3$ ). (D) Cumulative cell counts of MV-4-11 MN1wt and MN1null cells (mean $\pm S E M, n=3$ ). (E) Colony-forming cell (CFC) counts of MLL-AF9/Mn1wt and MLL-AF9/Mn1null cells (mean \pm SEM, $n=3$ ). (F) Morphology of representative CFC colonies of MLL-AF9/Mn1wt and MLL-AF9/Mn1null cells. Black scale bar represents $0.25 \mathrm{~mm}$. *P<0.05; **P<0.01; ns: not significant. 
Meningioma 1 regulates cell cycle, apoptosis and differentiation in MLL-AF9 cells

Next, we explored the impact of MN1 deletion on cell cycle, apoptosis and differentiation in MLL positive leukemic cell lines. Loss of MN1 inhibited cell cycle progression resulting in more cells in G0/G1 phase and fewer cells in $\mathrm{S}$ phase of the cell cycle compared to MN1wt cells (Figure 2A and Online Supplementary Figure S4A). In addi- tion, cell cycle progression was negatively associated with MN1 null cells by gene set enrichment analysis (GSEA) of gene expression profiling data (Online Supplementary Tables $S 4$ and $S 5$ and Online Supplementary Figures S4B and S5A$D)$. The proportion of Annexin $\mathrm{V}$ positive cells was significantly increased and the gene sets for apoptosis and the p53 pathway were highly enriched in MLL-AF9/Mn1null cells (Figure 2B, Online Supplementary Table 54 and Online
A

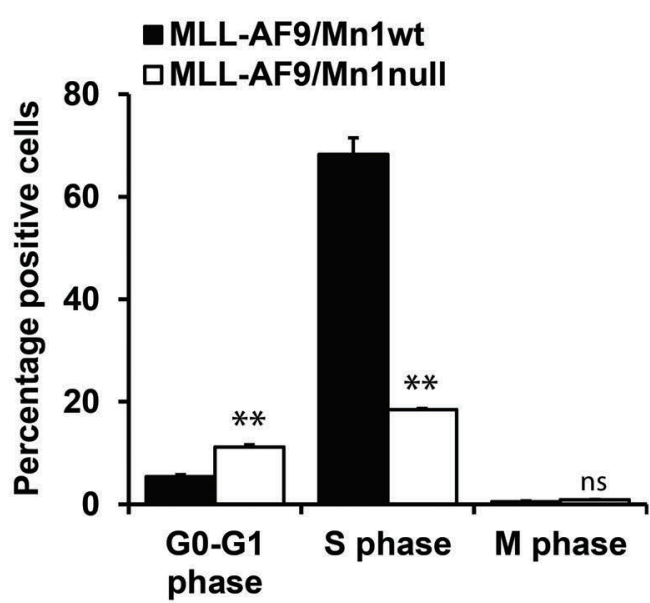

C

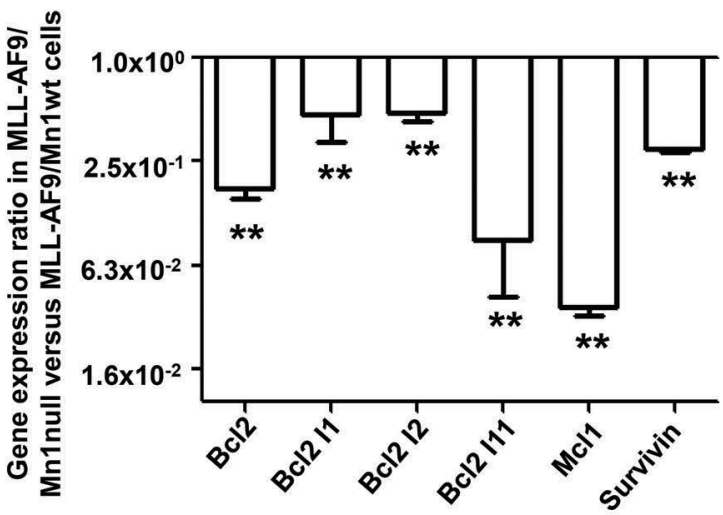

$\mathrm{E}$

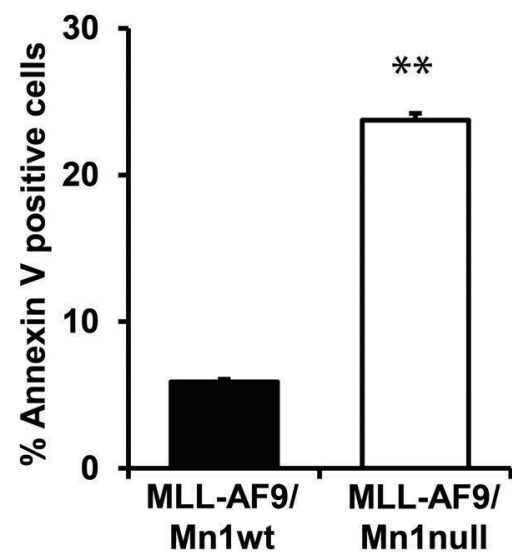

D

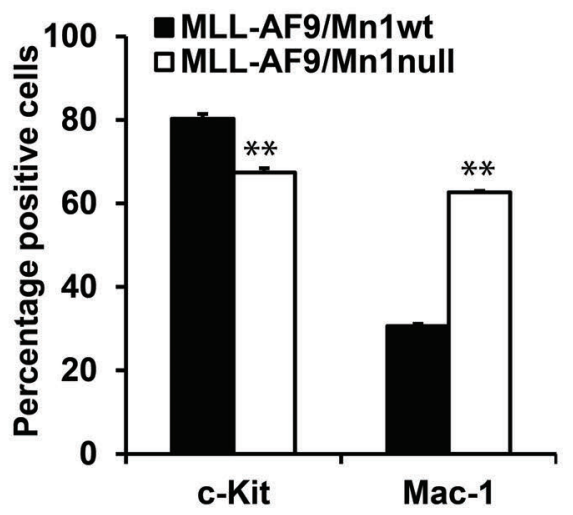

MLL-AF9/Mn1null

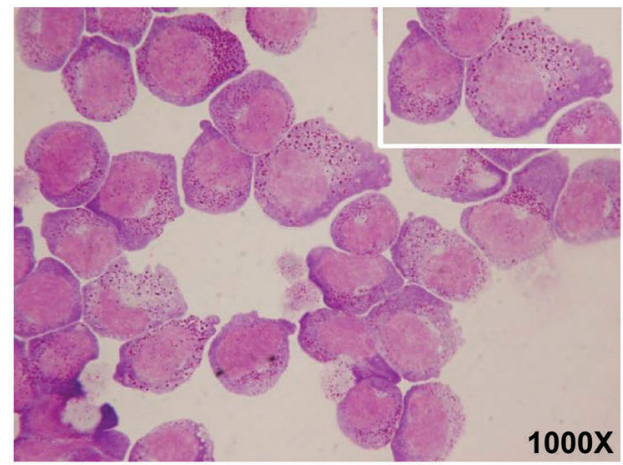

Figure 2. Meningioma 1 (MN1) regulates cell cycle, apoptosis and differentiation in MLL-AF9 cells. (A) Cell cycle analysis of MLL-AF9/Mn1wt and MLL-AF9/Mn1null cells [meantstandard error of mean (SEM), $n=3$ ]. (B) Proportion of apoptotic cells in MLL-AF9/Mn1wt and MLL-AF9/Mn1null cells (mean+SEM, $n=3$ ). (C) Relative gene expression of differentially expressed genes normalized to Abl1 in MLL-AF9/Mn1wt and MLL-AF9/Mn1null cells (mean \pm SEM, $n=3$ ). (D) Immunophenotype of in vitro cultured MLL-AF9/Mn1wt and MLL-AF9/Mn1 null cells (mean \pm SEM, $n=3$ ). (E) Morphology of in vitro cultured MLL-AF9/Mn1wt and MLL-AF9/Mn1null cells (original magnification 1,000-fold). $* P<0.05 ; * * P<0.01$; ns: not significant. 
Supplementary Figure S4E and $F$ ). In support of the GSEA analysis, a strong downregulation of the anti-apoptotic Bcl2 gene family, Mcl1 and Survivin was shown by qRTPCR (Figure 2C). MLL-AF9/Mn1null cells showed low levels of the hematopoietic stem/progenitor cell marker cKit/CD117 but high expression of the mature myeloid cell marker Mac1/CD11b and Gr-1/Ly-6G in Mn1 null cells compared to MLL-AF9/Mn1wt cells (Figure 2D and Online Supplementary Figure S6A). This observation was also supported by morphological analysis, as Mn1 null cells had a lower nuclear-cytoplasmic ratio and were more granulated (Figure 2E), and gene sets were enriched for myeloid and lymphoid cell differentiation (Online Supplementary Tables $S 4$ and S5, and Online Supplementary Figures S4B and S6B and C). MLL-AF9 oncogene expression was found to be unaltered or increased upon loss of Mn1 in MLLAF9/Mn1null cells when compared with MLLAF9/Mn1wt cells, excluding the possibility that downregulation of the oncogene is responsible for the antileukemic effects of MN1 loss (Online Supplementary Figure $S 7 A-C)$. Hence, MN1 deletion suppresses cell cycle, promotes apoptosis, and induces differentiation in MLL positive leukemia cells.

\section{MLL-AF9-induced leukemogenesis requires Meningioma 1}

To further investigate the role of Mn1 deletion in MLLAF9 leukemogenesis in vivo, we transplanted MLLAF9/Mn1wt and MLL-AF9/Mn1null cells in syngeneic mice and monitored onset of leukemia and survival. Mice transplanted with MLL-AF9/Mn1null cells had $<1 \%$ engraftment in all ten mice, whereas mice transplanted with MLL-AF9/Mn1wt cells showed $>90 \%$ engraftment at week 4 (Figure 3A). At eight weeks, engraftment in MLL-AF9/Mn1null was below 2\% (except for one mouse with $88 \%$ MLL-AF9 expressing cells, which consequently died), while 8 of 10 MLLAF9/Mn1wt mice died from leukemia and the two surviving mice showed $>90 \%$ engraftment (Figure $3 \mathrm{~A}$ ). Correspondingly, white blood cell (WBC) counts were significantly lower in MLL-AF9/Mn1null than in MLLAF9/Mn1wt mice (Figure 3B). Loss of Mn1 significantly prolonged survival as compared to Mn1wt mice (Figure 3C). At death, mice transplanted with MLL-AF9/Mn1 null cells had significantly lower spleen weight (Figure 3D). Since loss of Mn1 in MLL-AF9 cells did not show a potent engraftment ability, and to exclude the possibility of homing defects, we transplanted equal numbers of MLL-AF9 (Mn1wt/null) cells intravenously in sub-lethally irradiated mice. Mice were sacrificed after 8 and 24 hours and MLL-AF9/Mn1null cells showed better homing in bone marrow than mice transplanted with MLLAF9/Mn1wt cells, suggesting that differences in homing could not account for the differences in survival (Figure $3 \mathrm{E})$. In addition, to rule out the presence of any immunological effects against MLL-AF9/Mn1null cells, we transplanted MLL-AF9 (Mn1wt or null) cells in NSG mice (lacking cellular and humoral immunity) and monitored leukemia onset and survival. Similar to syngeneic mouse transplantation studies, MLL-AF9/Mn1null mice did not develop leukemia, while their MLLAF9/Mn1wt counterpart quickly died from leukemia (Online Supplementary Figures $S 8 A$ and $B$ and S9A-H for blood counts). Thus, MLL-AF9 positive murine leukemia requires MN1 expression to induce leukemia in vivo.

\section{MLL-AF9 and MLL-AF4 rearranged human leukemias also depend on Meningioma 1}

Besides syngeneic MLL-AF9 transplantation studies, we also assessed the role of MN1 deletion in MLL-AF4 (MV4-11) and MLL-AF9 (THP-1) leukemias in vivo. Equal numbers of THP-1 and MV-4-11 (MN1wt or MN1null) cells were transplanted intravenously in NSG mice, and the onset of leukemia and survival were assessed. MN1null cells showed lower engraftment of transplanted cells in peripheral blood at four weeks and improved blood counts compared to their wild-type counterparts (Figure 4A and B and Online Supplementary Figures S10 and S11). MN1 deletion significantly prolonged survival of mice transplanted with THP-1/MN1null and MV-4-11/MN1null clones (Figure 4C and D). At death, engraftment in bone marrow and spleen weight were also found lower in mice transplanted with MN1 deletion clones of THP-1 and MV4-11 (Figure 4E and F and Online Supplementary Figure S12A and $B$ ). In addition, we also evaluated the tumor-forming ability of THP-1/MN1null and MV-4-11/MN1null clones by subcutaneous transplantation in NOD-SCID mice. Tumor volumes were monitored every five days starting 15 days after transplantation. Deletion of MN1 led to significantly reduced tumor volumes for 2 of 3 MN1null clones as compared to wild-type MN1 human leukemic cell lines (Online Supplementary Figure S13A and B). Our in vivo transplantation studies suggest that loss of $\mathrm{MN1}$ critically affects leukemia proliferation in human MLLrearranged AML.

\section{Meningioma 1 overexpression restores leukemogenicity in MLL-AF9/Mn1null cells}

In an attempt to rescue the deleterious effects caused by Mn1 deletion, we over-expressed control (MIY) vector or MN1 in MLL-AF9/Mn1 null cells and characterized its properties in vitro and in vivo. MLL-AF9/Mn1null cells transduced with MN1 overcame the reduced proliferative capacity of MLL-AF9/Mn1null cells similarly to MLLAF9/Mn1wt cells (Figure 5A). Similarly, MLLAF9/Mn1null cells with MN1 expression restored the reduced colony-forming potential of MLL-AF9/Mn1null cells akin to MLL-AF9/Mn1wt cells (Figure 5B and C). We also studied the leukemogenic potential of MLLAF9/Mn1null cells rescued by MN1 expression in comparison with MLL-AF9 (Mn1wt or null) cells transduced with vector control. As previously mentioned in our manuscript, MLL-AF9/Mn1null cells do not possess the ability to engraft in mice. However, MLL-AF9/Mn1null cells with ectopic MN1 expression engrafted in mice and induced a leukemic phenotype with short survival similar to MLLAF9/Mn1wt mice (Figure 5D and $\mathrm{E}$ and Online Supplementary Figure S14). Hence, deleterious effects caused by loss of MN1 expression can be rescued by restoring the expression of MN1.

\section{Meningioma 1 maintains expression of the distal HOXA cluster and MEIS1}

MLL-AF9-mediated leukemogenesis is primarily mediated by upregulation of the Hox/Meis1 gene cluster and their target genes. ${ }^{33,35}$ DOT1L methylates histone H3 on lysine 79 (H3K79me2), which has been associated with MLL-AF9 binding and expression of Hoxa cluster genes and Meis1 in normal hematopoietic progenitors and MLL$r$ leukemias. ${ }^{25}$ Gene expression analysis by qRT-PCR in MLL-AF9/Mn1null and Mn1wt cells showed that Hoxa3 
through Hoxa7 were not significantly dysregulated, while Hoxa9, Hoxa10 and Meis1 were strongly down-regulated in MLL-AF9/Mn1null cells (Figure 6A). We also performed global gene expression analysis in MLL-AF9/Mn1wt versus MLL-AF9/Mn1null cells with significant differentially expressed genes (Online Supplementary Table S6 and Online Supplementary Figure S15A).

To evaluate a direct transcriptional effect of MLL-AF9 and MN1 on the distal Hoxa cluster genes, we analyzed the binding of MN1, MLL-AF9, Hoxa9, MEIS1 and dimethyl marks of H3K79 (representing DOT1L binding) at the Hoxa cluster locus from available chromatin immunoprecipitation-sequencing (ChIP-Seq) data sets.
MN1 chromatin marks were enriched at distal Hoxa cluster genes (Hoxa7 to Hoxa10) similar to MLL-AF9, MEIS1 and $\mathrm{H} 3 \mathrm{~K} 79 \mathrm{me} 2$ marks (Figure $6 \mathrm{~B}$ ), suggesting that MN1 is a direct regulator of Hoxa cluster gene expression. However, genome-wide co-localization of MLL-AF9 and MN1 chromatin did not show any overlap of a significant number of chromatin marks, in contrast to the high overlap of MN1 with Hoxa9 and MEIS1 (Figure 6C-E), suggesting that $\mathrm{MN1}$ is essential for MLL-AF9 primarily due to its role as a co-factor of Hoxa9 and Meis1.

To substantiate this observation, we evaluated the expression of $\mathrm{Bcl} 2$ in MLL-AF9/Mn1 wild-type and null cells, as Bcl2 is a known target of Hoxa9 but not of MLL-
A

MLL-AF9/Mn1wt 口MLL-AF9/Mn1null

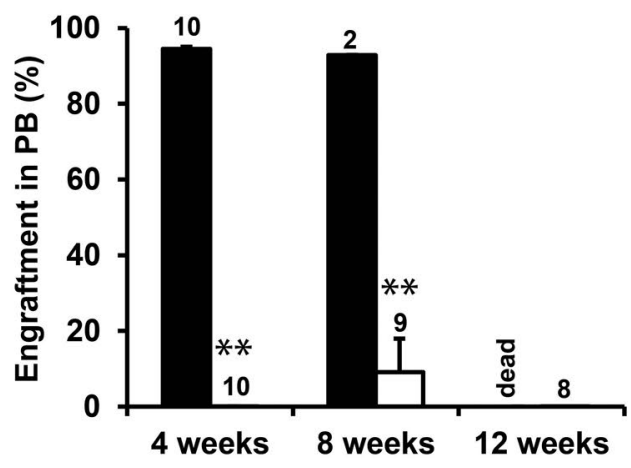

C

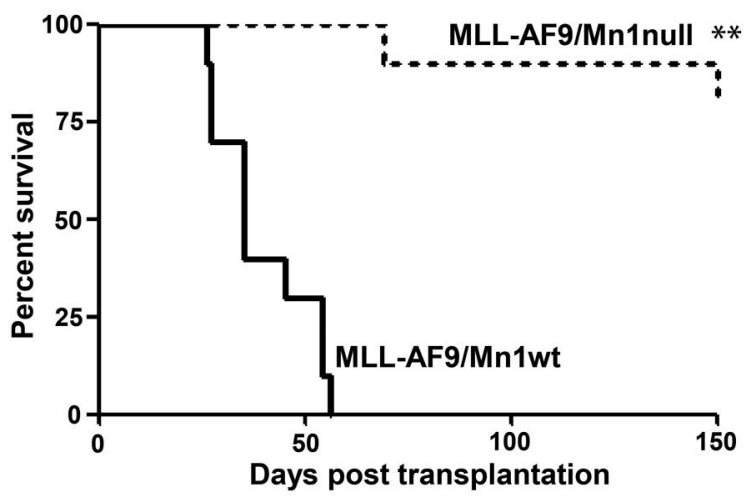

E

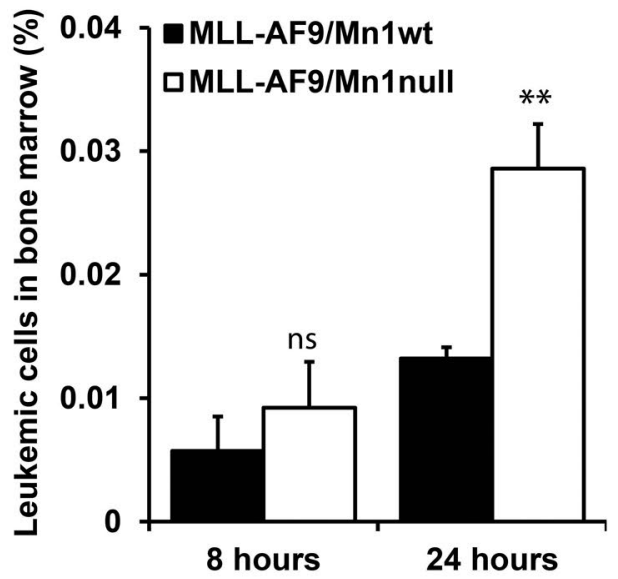

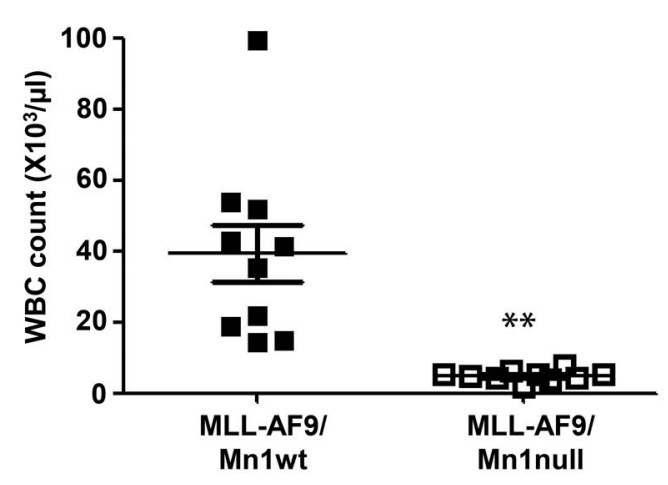

D

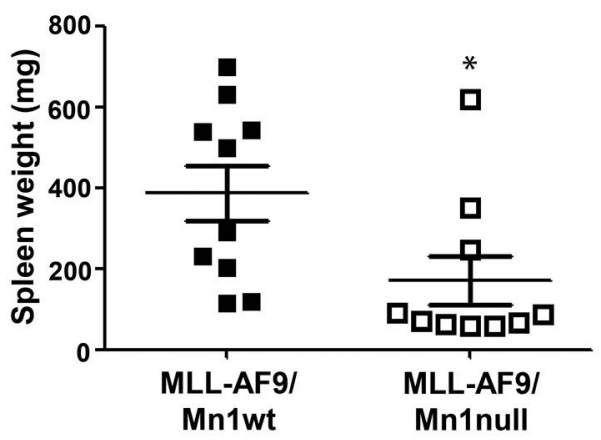

Figure 3. MLL-AF9-induced leukemogenesis requires Meningioma 1 (MN1). (A) Engraftment of MLL-AF9/Mn1wt and MLL-AF9/Mn1null cells in peripheral blood $(\mathrm{PB})$ at the indicated time points [mean \pm standard error of mean (SEM) of the indicated number of mice]. (B) White blood cell count (WBC) in peripheral blood of mice at four weeks. Mice received transplants of MLL-AF9/Mn1wt and MLL-AF9/Mn1null cells (mean+SEM, $n=10$ ). (C) Survival of mice receiving transplants of MLL-AF9/Mn1wt and MLLAF9/Mn1null cells (log rank test). (D) Spleen weight of the indicated mice at the time of death (mean $\pm S E M, n=10$ ). ( $E$ ) Homing assay. Percentage of MLL-AF9/Mn1wt and MLL-AF9/Mn1null cells in bone marrow at 8 and 24 hours after transplantation (mean \pm SEM of the number of mice, $n=5$ for each cell type and each time point). ${ }^{\star} P<0.05$; $* * P<0.01$; ns: not significant. 
AF9 (Figure 6F). ${ }^{36}$ In agreement with this, $\mathrm{Bcl} 2$ and related genes were strongly down-regulated upon deletion of Mn1 in MLL-AF9 cells (Figure 2C), confirming the critical role of $\mathrm{MN1}$ in Hoxa9/Meis1 regulated gene expression. We also identified putative Hoxa9/Hoxa10 targets differentially expressed and regulated in our gene expression data and GSEA analysis in MLL-AF9 (Mn1wt vs. Mn1null) cells (Online Supplementary Table S7 and Online Supplementary Figure S15B and C). Significantly, more Hoxa9/Hoxa10 target genes were down-regulated in MLL-AF9/Mn1null ( $P=0.027$, from hypergeometric distribution). Thus, our data suggest that MN1 is required in MLL-r leukemia to
A
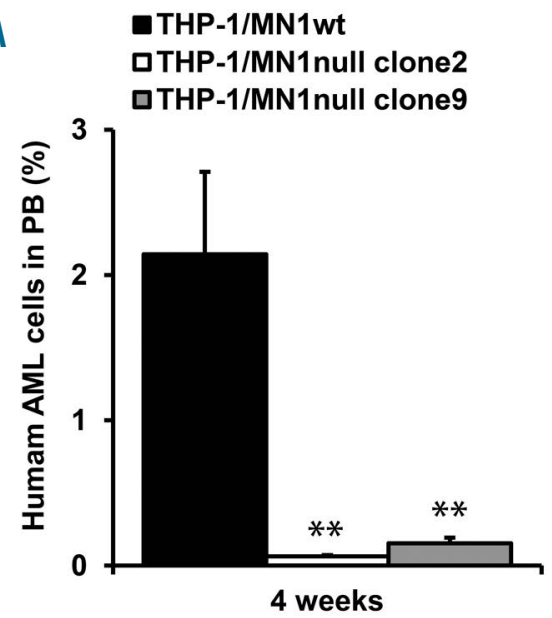

C

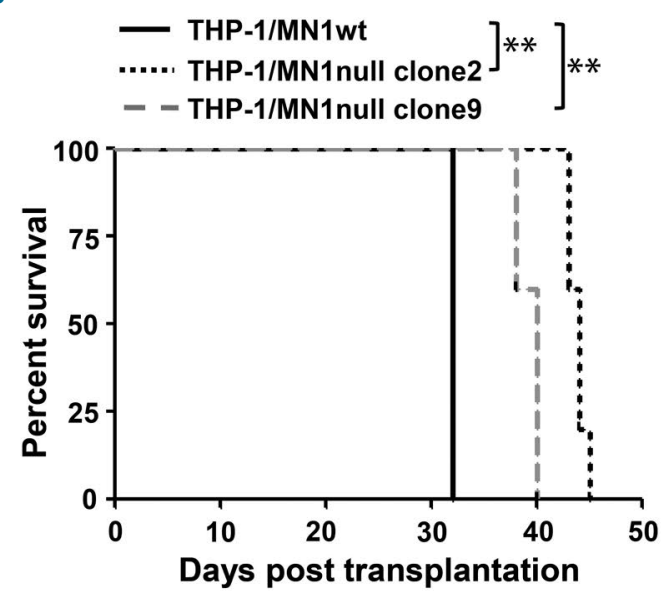

$E$

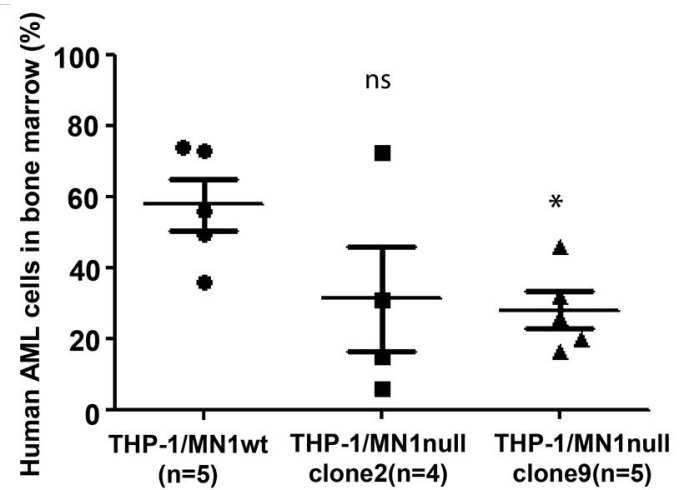

B

-MV-4-11/MN1wt

口MV-4-11/MN1null clone1

口MV-4-11/MN1null clone10

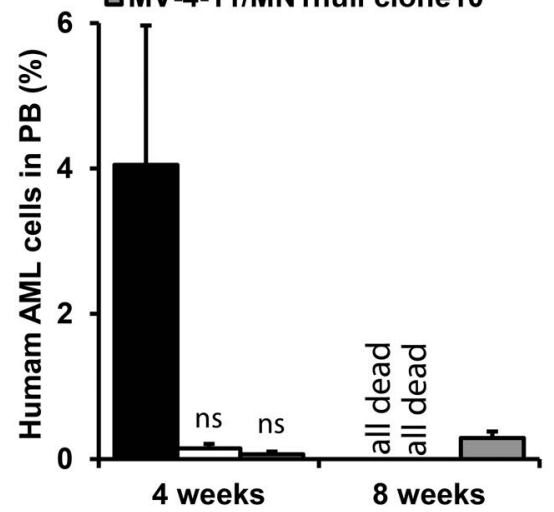

D
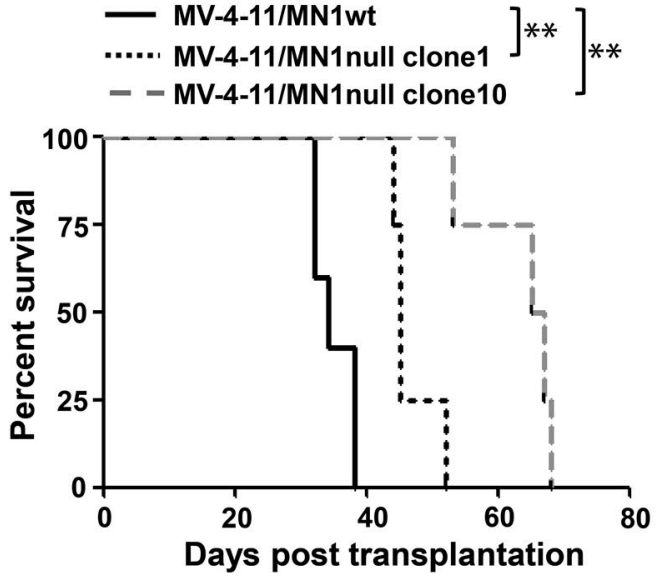

$\mathrm{F}$

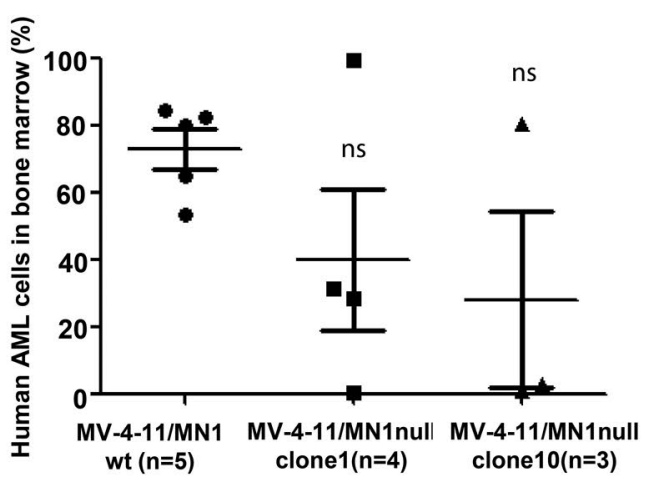

Figure 4. MLL-AF9 and MLL-AF4 rearranged human leukemias also depend on Meningioma 1 (MN1). (A) Engraftment of THP-1/MN1wt and THP-1/MN1null cells in peripheral blood (PB) at four weeks [mean+standard error of mean (SEM), $n=5$ for THP-1/MN1wt. THP-1/MN1null clone2 and 9]. (B) Engraftment of MV-411/MN1wt and MV-4-11/MN1null cells in peripheral blood (PB) at 4 and 8 weeks (mean \pm SEM; $n=5$ for MV-4-11/MN1wt, $n=4$ for MV-4-11/MN1null clone1 and 10). (C) Survival of mice receiving transplants of THP-1/MN1wt and THP-1/MN1null cells (log rank test); ( $n=5$ for THP-1/MN1wt, THP-1/MN1null clone2 and 9). (D) Survival of mice receiving transplants of MV-4-11/MN1wt and MV-4-11/MN1null cells (log rank test); ( $\mathrm{n}=5$ for MV-4-11/MN1wt, $n=4$ for MV-4-11/MN1null clone1 and 10). (E) Percentage of THP-1 (MN1wt or MN1null clone) cells in bone marrow at death (mean $+S E M$; $n=5$ for THP-1/MN1wt and THP-1/MN1null clone9, $n=4$ for THP-1/MN1null clone2). (F) Percentage of MV-4-11 (MN1wt or MN1null clone) cells in bone marrow at death (mean \pm SEM; $n=5$ for MV-4-11/MN1wt, $n=4$ for MV-411/MN1null clone1 and $n=3$ for MV-4-11/MN1null clone10). AML: acute myeloid leukemia; ** $P<0.01$; ns: not significant. 
maintain expression of the distal Hoxa cluster and Meis1 and as a co-factor of the Hoxa9/Meis1 transcriptional complex and their target genes, which also includes MLL.

Meningioma 1 as therapeutic target in MLL-AF9 acute myeloid leukemia

To evaluate $\mathrm{MN1}$ as a therapeutic target in MLL positive AML, we tested the effect of MN1 siRNA in primary human AML cells. As a proof of principle, we first tested MN1 knockdown using LNP packaged with anti-MN1 siRNA in the ME-1 cell line, which expresses MN1 at high levels. MN1 expression was reduced up to 4-fold in antiMN1 siRNA treated cells as compared to control siRNA treated cells (Online Supplementary Figure S16A). Furthermore, we evaluated the effect of MN1 knockdown in $\mathrm{CD}_{3} 4^{+}$hematopoietic progenitor cells from four
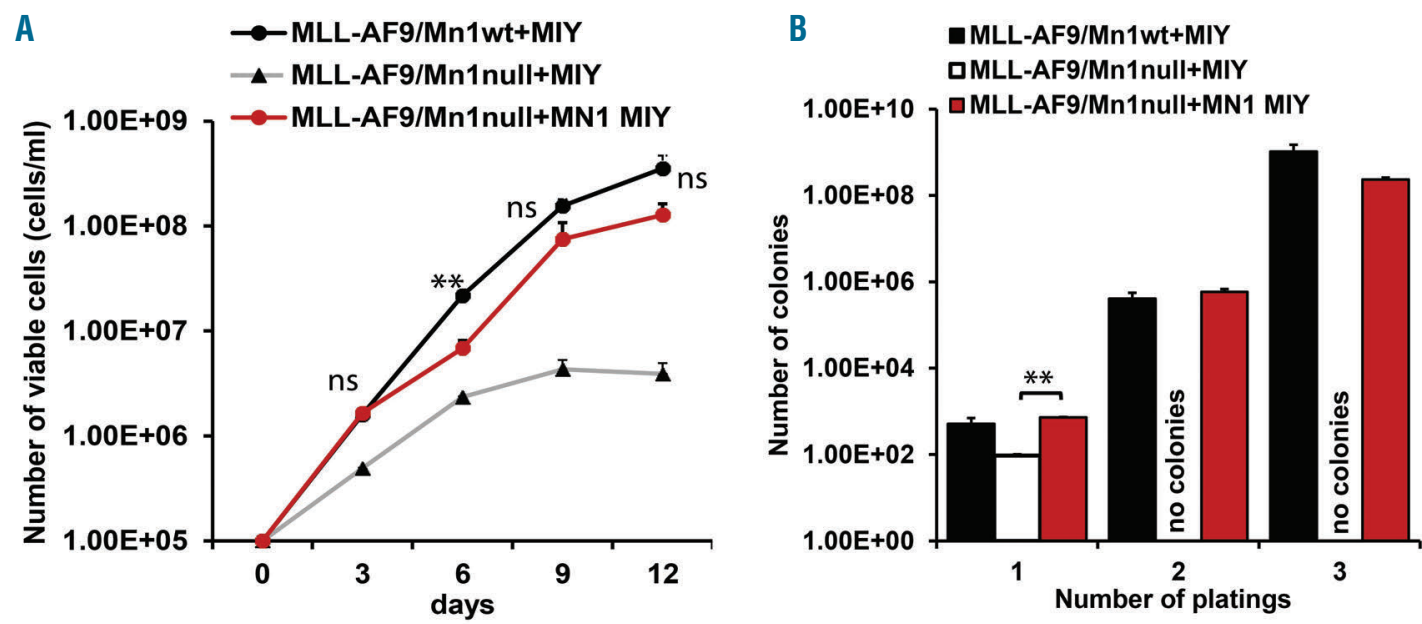

C
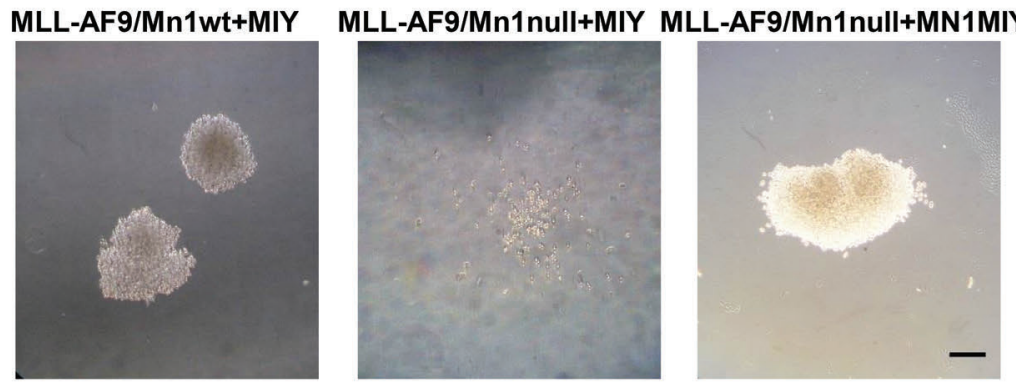

D

MLL-AF9/Mn1wt+MIY

口MLL-AF9/Mn1null+MIY 口MLL-AF9/Mn1null+MN1MIY

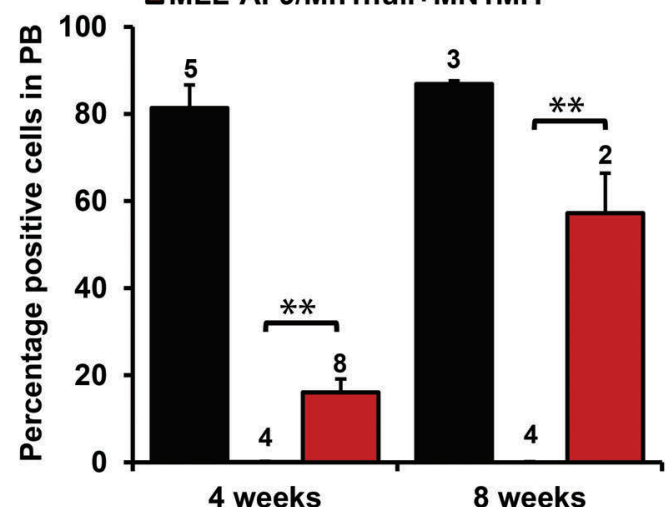

E
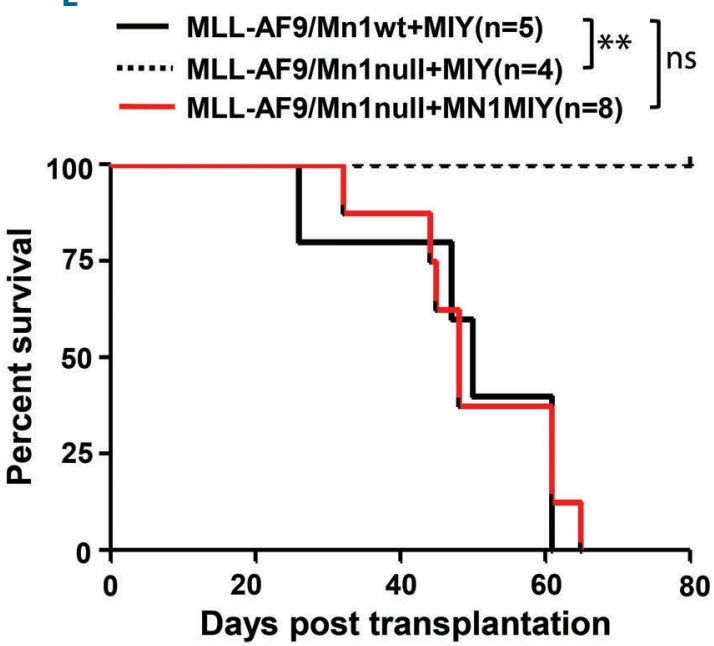

Figure 5. Meningioma 1 (MN1) overexpression restores leukemogenicity in MLL-AF9/Mn1null cells. (A) Cumulative cell counts of MLL-AF9/Mn1wt and MLL AF9/Mn1null cells transduced with control or MN1MIY plasmid [mean \pm standard error of mean (SEM), $n=3$ ]. (B) Colony-forming cell (CFC) counts of MLL-AF9/Mn1wt and MLL-AF9/Mn1null cells transduced with control or MN1MIY plasmid (mean \pm SEM, $n=3$ ). (C) Morphology of representative CFC colonies of MLL-AF9/Mn1wt and MLL-AF9/Mn1null cells transduced with control or MN1MIY plasmid. Black scale bar represents 0.25 mm. (D) Engraftment of MLL-AF9/Mn1wt and MLL-AF9/Mn1null cells transduced with control or MN1MIY plasmid in peripheral blood (PB) at 4 and 8 weeks after transplantation (mean \pm SEM of the indicated number of mice). (E) Survival of mice receiving transplants of MLL-AF9/Mn1wt and MLL-AF9/Mn1null cells transduced with control or MN1MIY plasmid (log-rank test). **P<0.01; ns: not significant. 
healthy stem cell donors and five MLL-AF9 positive AML patients in colony forming cell (CFC) assays. Colony numbers and size were significantly reduced in MLL-AF9 positive AML cells treated with anti-MN1 siRNA compared to $\mathrm{CD} 34^{+}$cells from healthy donors when normalized to control siRNA treated cells (Figure 7A and B). Cell numbers from CFC assays were also found significantly reduced in MLL-AF9 positive AML cells treated with antiMN1 siRNA (Figure 7C). In order to exclude toxic effects of the LNP/siRNA formulation, we tested an additional anti-MN1 siRNA (MN1 siRNA-3) and found similar specific inhibitory effects with anti-MN1 siRNA-3 against MLL-AF9 positive primary human AML cells (Online Supplementary Figure $S 16 B$ and C). Thus, MN1 expression is critical for proliferation of MLL-transformed leukemic cells while it has no effect on $\mathrm{CD} 4^{+}$cells from healthy donors, suggesting $\mathrm{MN1}$ as a therapeutic target in MLLtransformed AML.

B

A
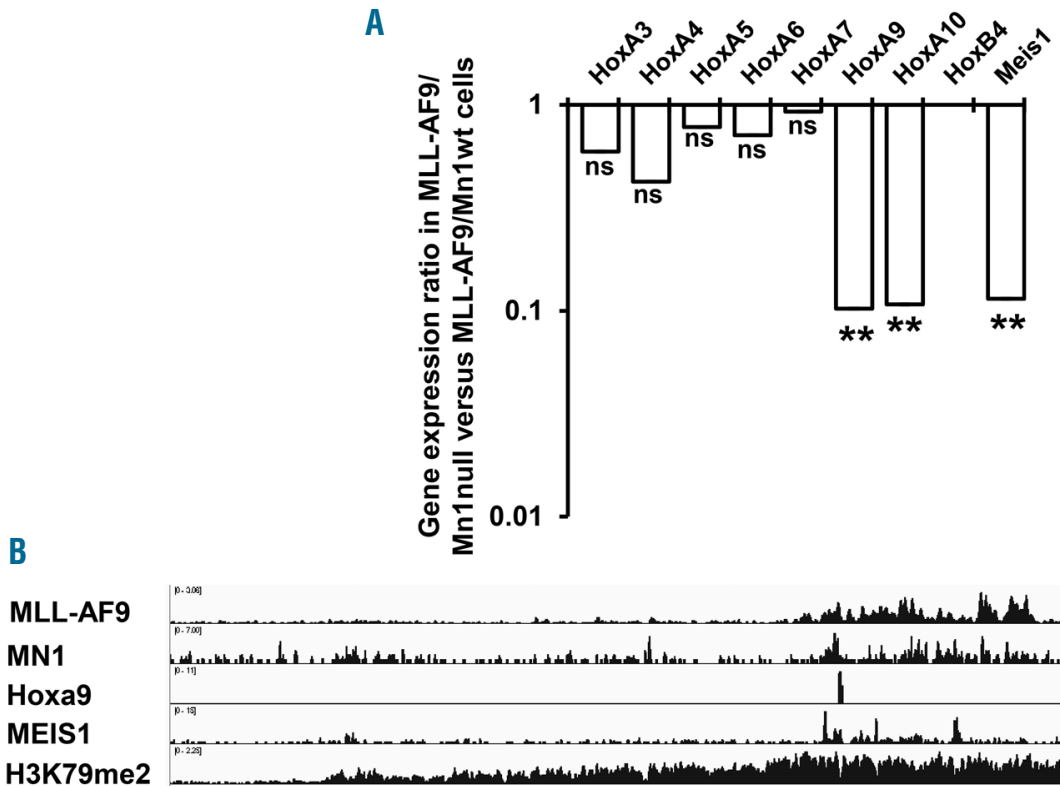

H3K79me2
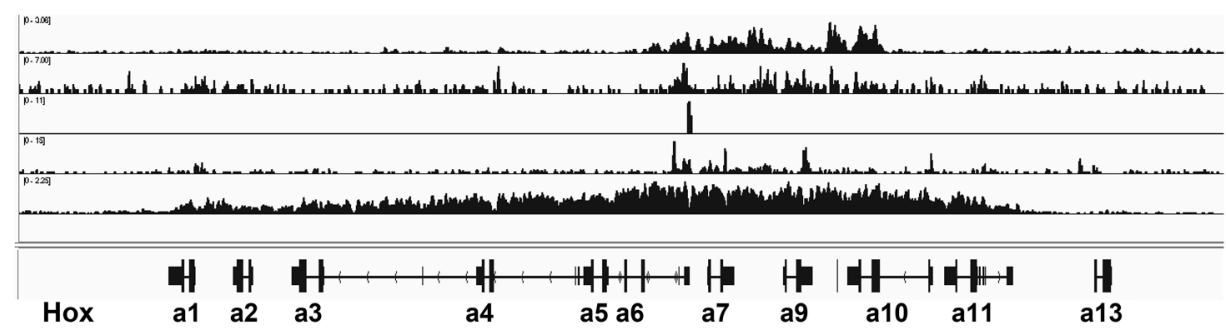

C

D

$\mathbf{E}$
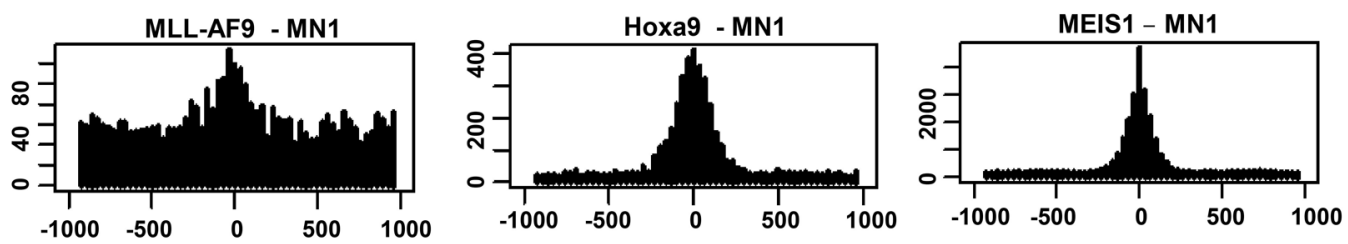

F

MLL-AF9

MN1

Hoxa9

MEIS1

H3K79me2

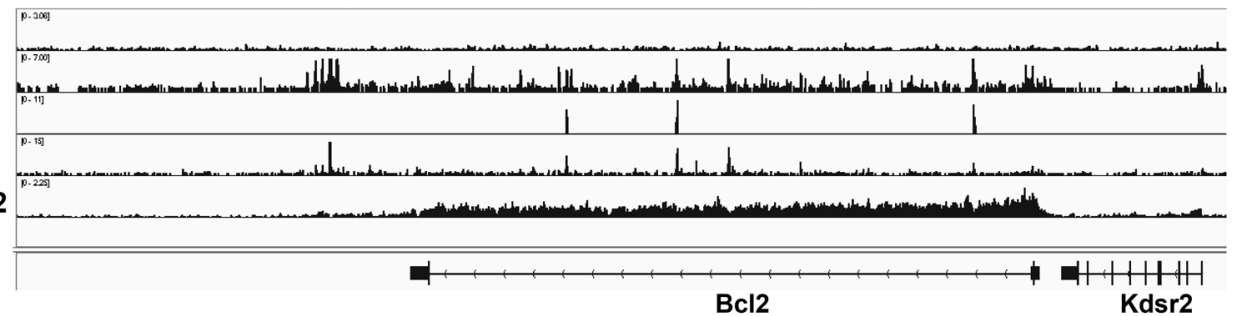

Figure 6. Mn1 maintains distal Hoxa cluster and Meis1 expression. (A) Relative gene expression of differentially expressed genes normalized to Abl1 in MLLAF9/Mn1wt andMLL-AF9/Mn1null murine cells [mean \pm standard error of mean (SEM), n=3]. (B) Chromatin immunoprecipitation-sequencing (ChIP-Seq) for MLL-AF9, MN1, Hoxa9, MEIS1 and H3K79me2 in different leukemic cell lines (details in Methods). Screen shot of the Hoxa cluster with binding peaks of the above mentioned proteins. (C) Histogram showing the distance between peak maxima of MLL-AF9 peaks to their closest MN1 peak, indicating that MN1-binding sites are less frequently enriched at MLL-AF9-binding sites. (D) Histogram showing the distance between peak maxima of Hoxa9 peaks to their closest MN1 peak, indicating that MN1binding sites are highly enriched at Hoxa9-binding sites. (E) Histogram showing the distance between peak maxima of MEIS1 peaks to their closest MN1 peak, indicating that MN1-binding sites are highly enriched at MEIS1-binding sites. (F) ChIP-Seq for MLL-AF9, MN1, Hoxa9, MEIS1 and H3K79me2 in different leukemic cell lines (details in Methods). A screen shot of the Bcl2 gene with binding peaks of the aforementioned proteins. ${ }^{\star} P<0.05$; ${ }^{*} P<0.01$; ns: not significant. 


\section{Discussion}

Acute myeloid leukemia pathogenesis requires a multitude of genetic and epigenetic alterations for its initiation and progression. ${ }^{1}$ One such genetic alteration is high expression of $\mathrm{MN1}$, which is widely reported as an independent poor prognostic marker in AML patients, and its induced overexpression in mouse cells induces aggressive AML.- ${ }^{7-9,12,13,23}$ In the knockout studies of MN1, mice died young from cleft palate and severe defects in development
A

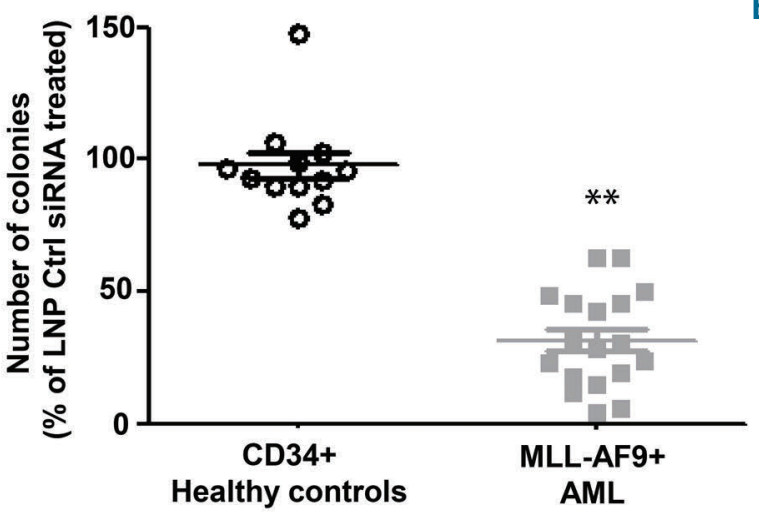

C

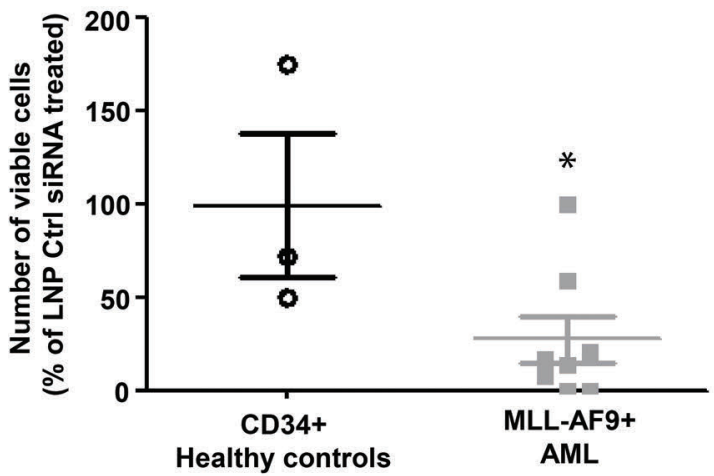

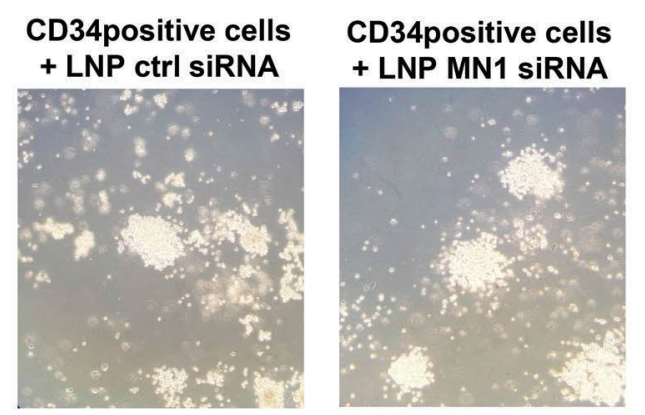

MLL-AF9 positive AML MLL-AF9 positive AML + LNP Ctrl siRNA

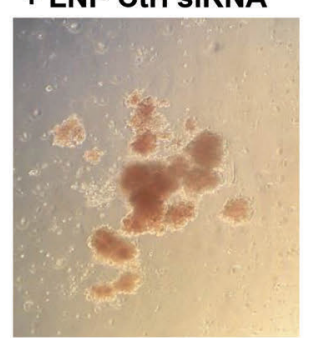

\section{+ LNP MN1 siRNA}

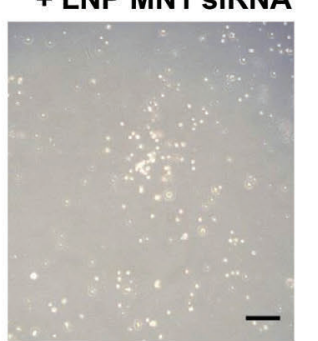

D

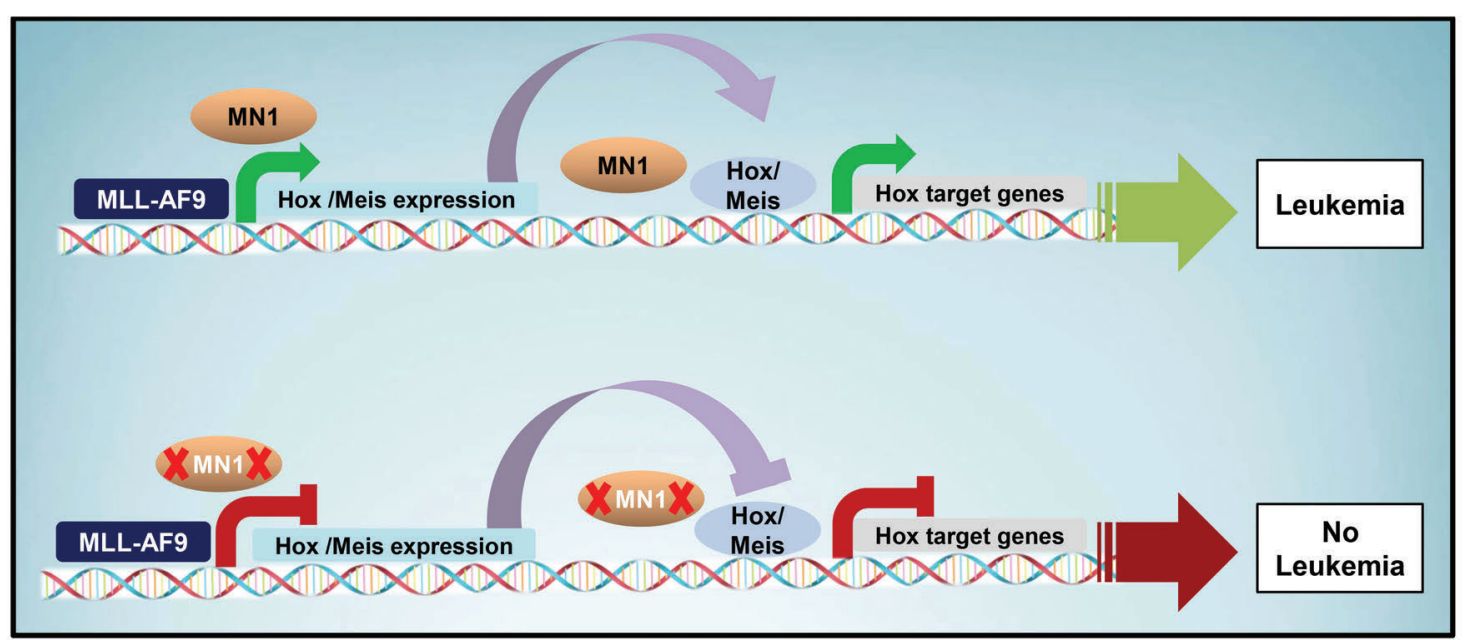

Figure 7. Meningioma 1 (MN1) as therapeutic target in MLL-AF9 acute myeloid leukemia (AML). (A) Colony-forming cell (CFC) counts of CD34+ healthy controls (Ctrl) and MLL-AF9 positive AML patient cells treated with anti-MN1 siRNA/LNP formulation normalized to control siRNA/lipid nanoparticle (LNP) formulation [mean \pm standard error of mean (SEM), cells from $n=4$ CD34 ${ }^{+}$healthy donors with 3 repeats, and cells from $n=4$ MLL-AF9 positive AML patients with 4 repeats and 2 repeats from 1 patient]. (B) Morphology of representative CFC colonies of CD34+ healthy controls and MLL-AF9 positive AML patient cells treated with control or anti-MN1 siRNA/LNP formulation. Black scale bar represents $0.25 \mathrm{~mm}$. (C) Number of viable cells from colonies of CD34 $4^{+}$healthy controls and MLL-AF9 positive AML patient cells treated with anti-MN1 siRNA/LNP formulation normalized to control siRNA/LNP treated cells (mean \pm SEM, cells from $n=1$ CD34 ${ }^{+}$healthy donor in triplicate, and cells from $n=4$ MLL-AF9 positive AML patients in duplicate from each patient). ${ }^{*}<0.05$; $* * P<0.01$; ns: not significant. (D) Model of MN1-dependency in MLLrearranged AML. MLL-AF9 and MN1 induces expression of HOXA cluster genes and MEIS1. MN1 is a critical co-factor of the HOXA9/MEIS1 transcriptional complex. Loss of MN1 affects HOXA expression and renders the HOXA9/MEIS1 complex inactive, and loss of this critical downstream complex of MLL-AF9 blocks its transforming ability. 
of various bones in the skull. In addition, MN1 KO mice apparently did not develop hematopoietic defects during the first few weeks of life until they died, suggesting that MN1 inhibition or deletion has a therapeutic window when targeted in leukemic cells. ${ }^{28}$ It has been previously reported that transient $M N 1$ knockdown via siRNA in human leukemia cell lines reduces their proliferation; ${ }^{20}$ however, perpetual loss of $M N 1$ expression has not been comprehensively studied in murine or human leukemias. Our study shows that CRISPR-Cas-mediated loss of MN1 substantially impairs leukemogenesis in MLL-r leukemias. $\mathrm{MN} 1$ loss prevents in vitro proliferation and colony-forming potential of MLL-AF9 and MLL-AF4-dependent cells. Correspondingly, we demonstrate that MN1 inactivation inhibits cell cycle, promotes apoptosis and induces differentiation in MLL-r leukemic cells. In addition, loss of MN1 significantly impairs MLL-AF9-mediated murine leukemogenesis and reduces leukemic and tumor growth of human MLL-rearranged leukemias in vivo. Our results are supported by a previous study, which found that $\mathrm{Mn} 1 \mathrm{co}-$ operated with Mll-AF9 in leukemogenesis in an in vivo transplantation assay. ${ }^{6}$ The homing potential of MLLAF9/Mn1null cells is not affected by Mn1 deletion. We also show that re-expression of MN1 in MLLAF9/Mn1null cells restores the leukemic potential of MLLAF9.

Both MN1 and MLL-AF9-induced leukemias rely on expression of the Hoxa9/Meis1 complex. ${ }^{2,25,33,35,37,38}$ On a genome-wide level chromatin binding of MN1 largely over-lapped with HOXA9 and MEIS1 binding, suggesting that the previously described function of MN1 as a co-factor of HOXA9/MEIS1 is its major contribution to the leukemogenic activity of MLL-AF9, as illustrated in our study (Figure 7D). This is supported by our gene expression data showing that HOXA9 target genes are primarily down-regulated upon MN1 deletion. Previous studies have found that both MLL-AF9 and MN1-induced leukemias are dependent on Dot 11.14,25,26 MLL fusions misdirect DOT1L to the promoters of Hoxa cluster genes and Meis1 leading to H3K79 methylation and constitutive activation of these genes. ${ }^{2,25,33}$ It is important to note that MN1 transformed HSC have high expression of HOXA9 irrespective of DOT1L expression. ${ }^{14}$ In support of this observation, we show that loss of $\mathrm{MN1}$ can overcome the effect of MLL-AF9 and DOT1L-directed dysregulation of HOXA cluster genes and MEIS1.

The leukemogenicity of MLL-positive leukemias is dependent on genes such as the Mll wild-type allele, AF9, Dot11, JMJD1C, PU.1, CBX8, Hoxa7, Hoxa9 and S6K1, which mediate the leukemogenic activity of MLL-AF9 predominantly by Dot1l gene activation. ${ }^{26,33,37-41}$ Our data provide additional insights into MLL-rearranged leukemia through the addition of MN1 as a crucial co-factor regulating the HOXA cluster, MEIS1 and their target genes. As U937 cells were moderately affected by MN1 deletion, we speculate that cells with high HOXA9/MEIS1 expression may also depend on MN1 like MLL-r cells. Therefore, we predict that $\mathrm{MN1}$ is not only required for MLL-transformed leukemias, but for approximately $50 \%$ of $\mathrm{AML}$ cases, which show dysregulated expression of the clustered homeobox genes.

High MN1 expression has been reported as a poor prognostic marker in AML patients, and its overexpression induces ATRA resistance and AML in mouse cells. ${ }^{7-9,12}$ ${ }^{13,23} \mathrm{Also}$, it is most highly expressed in CMP, and scantily expressed in HSC and more differentiated cells. ${ }^{23}$ Interestingly, induced MN1 expression cannot transform stem cells, as previously described, ${ }^{23}$ and we hereby report that proliferation of $\mathrm{CD}_{3} 4^{+}$cells remain unaffected by depletion of MN1 expression. Hence, the expression characteristics of $\mathrm{MN1}$ seem to be favorable for therapeutic targeting. A potential therapeutic index is indicated by lack of an inhibitory effect in normal stem/progenitor cells and a specific inhibitory effect in AML cells. Therefore, targeting MN1 by LNP/siRNA formulations holds a strong potential therapeutic avenue, as suggested by our previous proof-of-principle study in a PDX model of acute lymphoblastic leukemia in vivo. ${ }^{32}$

In summary, we identify $\mathrm{MN1}$ as an essential protein in MLL fusion gene leukemia in vivo and highlight its importance as a co-factor of the HOXA9/MEIS1 complex. An LNP/siRNA formulation against MN1 effectively inhibits the colony-forming potential of MLL-r primary AML cells, suggesting that MN1 is a valid and druggable target in AML.

\section{Acknowledgments}

We thank Silke Glowotz, Annett Reinsch, Basem Othman, Kerstin Goerlich, Martin Wichmann, Nadine Kattre and Renate Schottmann for technical help. We also thank Dr. Matthias Ballmaier and the staff of the Cell Sorting Core Facility of Hannover Medical School for their excellent service (supported in part by the Braukmann-Wittenberg-Herz-Stiftung).

\section{Funding}

This study was supported by the Rudolf-Bartling Stiftung, an ERC grant under the European Union's Horizon 2020 research and innovation program (n. 638035), Deutsche Krebshilfe (70112697) and DFG grants HE 5240/5-1, HE 5240/5-2, HE 5240/6-1 and HE 5240/6-2.

\section{References}

1. Papaemmanuil E, Gerstung M, Bullinger L, et al. Genomic Classification and Prognosis in Acute Myeloid Leukemia. N Engl J Med. 2016;374(23):2209-2221.

2. Slany RK. The molecular mechanics of mixed lineage leukemia. Oncogene. 2016;35(40):5215-5223.

3. Meyer C, Burmeister T, Groger D, et al. The MLL recombinome of acute leukemias in 2017. Leukemia. 2018;32(2):273-284

4. Milne TA. Mouse models of MLL leukemia: recapitulating the human disease. Blood. 2017;129(16):2217-2223.

5. Mueller D, Garcia-Cuellar MP, Bach C, Buhl S, Maethner E, Slany RK. Misguided transcriptional elongation causes mixed lineage leukemia. PLoS Biol. 2009; 7(11):e1000249.

6. Bergerson RJ, Collier LS, Sarver AL, et al. An insertional mutagenesis screen identi- fies genes that cooperate with Mll-AF9 in a murine leukemogenesis model. Blood. 2012;119(19):4512-4523.

7. Heuser M, Argiropoulos B, Kuchenbauer F, et al. MN1 overexpression induces acute myeloid leukemia in mice and predicts ATRA resistance in patients with AML. Blood. 2007;110(5):1639-1647.

8. Heuser M, Beutel G, Krauter J, et al. High meningioma 1 (MN1) expression as a predictor for poor outcome in acute myeloid 
